\title{
Functional reprogramming of monocytes in acute and convalescent severe COVID-19 patients
}

\section{Elisa Brauns}

Université Libre de Bruxelles

Abdulkader Azouz

Université Libre de Bruxelles https://orcid.org/0000-0001-8626-4370

\section{David Grimaldi}

Hôpital Erasme

Hanxi Xiao

University of Pittsburgh

Séverine Thomas

Université Libre de Bruxelles

Muriel Nguyen

Université Libre de Bruxelles

Véronique Olislagers

Université Libre de Bruxelles

Ines Vu Duc

Université Libre de Bruxelles

Carmen Orte Cano

Höpital Erasme

Véronique Del Marmol

Höpital Erasme

Pieter Pannus

Sciensano

\section{Frédérick Libert}

Université Libre de Bruxelles

\section{Sven Saussez}

University of Mons https://orcid.org/0000-0002-3655-1854

Nicolas Dauby

CHU Saint-Pierre

Jishnu Das

University of Pittsburgh School of Medicine https://orcid.org/0000-0002-5747-064X

Arnaud Marchant

ULB

Stanislas Goriely ( $\square$ stanislas.goriely@ulb.be ) 


\section{Article}

Keywords: Myeloid Compartment Dysregulation, Immune Alteration, Cytokine Responses, Chromatin Accessibility

Posted Date: July 31st, 2021

DOl: https://doi.org/10.21203/rs.3.rs-766032/v1

License: (c) (i) This work is licensed under a Creative Commons Attribution 4.0 International License. Read Full License

Version of Record: A version of this preprint was published at JCI Insight on April 5th, 2022. See the published version at https://doi.org/10.1172/jci.insight.154183. 


\section{Functional reprogramming of monocytes in acute and convalescent severe COVID-19 patients}

Elisa Brauns ${ }^{1}$, Abdulkader Azouz ${ }^{1}$, David Grimaldi ${ }^{2}$, Hanxi Xiao ${ }^{3}$, Séverine Thomas ${ }^{1}$, Muriel Nguyen ${ }^{1}$, Véronique Olislagers ${ }^{1}$, Ines $\mathrm{Vu} \mathrm{Duc}^{1}$, Carmen Orte Cano ${ }^{4}$, Véronique Del Marmol ${ }^{4}$, Pieter Pannus ${ }^{5}$, Frédérick Libert ${ }^{6}$, Sven Saussez ${ }^{7}$, Nicolas Dauby ${ }^{1,8}$, Jishnu Das ${ }^{3}$, Arnaud Marchant ${ }^{1}$, Stanislas Goriely ${ }^{1}$

\section{Affiliations}

1. Institute for medical Immunology (IMI) and ULB Center for Research in Immunology (UCRI), ULB, Brussels, Belgium

2. Intensive Care Unit, Hôpital Erasme, Brussels, Belgium

3. Center for Systems Immunology, Department of Immunology, University of Pittsburgh School of Medicine; Department of Computational and Systems Biology, University of Pittsburgh School of Medicine, USA

4. Department of Dermatology, Hôpital Erasme, Brussels, Belgium

5. SD Epidemiology and Public Health, Sciensano, Anderlecht, Belgium

6. Institute of Interdisciplinary Research (IRIBHM) and Brightcore, ULB, Brussels, Belgium

7. Department of Otolaryngology, Epicura, Mons, Belgium

8. Department of Infectious diseases, Hôpital Saint-Pierre, Brussels, Belgium

\section{Lead contact:}

S. GORIELY, Institute for Medical Immunology, 8 rue Adrienne Bolland, B-6041

Charleroi-Gosselies, Belgium; Phone: 32-2-650-9588; Fax: 32-2-650-9563; E-mail: stanislas.goriely@ulb.be 


\section{Abstract}

Severe COVID-19 disease is associated with dysregulation of the myeloid compartment during acute infection. Survivors frequently experience long-lasting sequelae but little is known about the eventual persistence of this immune alteration. Herein, we evaluated Toll-like receptor-induced cytokine responses in a cohort of mild to critical patients during acute or convalescent phases $(n=97)$. In the acute phase, we observed impaired cytokine production by monocytes in the most severe patients. This capacity was globally restored in convalescent patients. Yet, we observed increased responsiveness to TLR1/2 ligation in patients that recovered from severe disease, indicating that these cells display distinct functional properties at the different stages of the disease. We identified a specific transcriptomic and epigenomic state in monocytes from acute severe patients that can account for their functional refractoriness. The molecular profile of monocytes from recovering patients was distinct and characterized by increased chromatin accessibility at AP-1 and MAF loci. These results demonstrate that severe COVID-19 infection has a profound impact on the differentiation status and function of circulating monocytes both during the acute and the convalescent phases in a completely distinct manner. This could have important implications for our understanding of short and long-term COVID19-related morbidity. 


\section{Introduction}

COVID-19, caused by the SARS-CoV-2 virus primarily infects the respiratory tract. There is a very broad spectrum of disease presentation. Some people who get COVID-19 have only mild symptoms. But for others, infection leads to pneumonia, respiratory failure, and, in some cases, death. The major complication of severe COVID-19 infection is acute respiratory distress syndrome (ARDS) presenting with dyspnoea and acute respiratory failure that requires mechanical ventilation. Severe COVID-19 appears to be associated with coagulopathy presenting as thrombosis in various organs and it is proposed that SARS-CoV2 causes lesions to endothelial cells which then triggers an inflammation throughout the body and fuels ARDS $(1,2)$. Multiple studies indicate that the mononuclear phagocyte system is strongly perturbed during acute infection and contributes to this hyperinflammatory complications (3-5). In patients with mild COVID-19, there is a specific increase of inflammatory monocytes that display a strong interferon (IFN)-stimulated gene signature. This was shown to be absent in severe disease. Instead, in these patients, there are signs of emergency myelopoiesis, marked by the occurrence of immunosuppressive neutrophils and HLA-DR ${ }^{L 0}$ monocytes (6). Impaired type I IFN production and decreased expression of HLADR by plasmacytoid and conventional dendritic cells (DCs), respectively, was also reported in these patients $(7,8)$.

In view of the novel nature of this disease, little is known about the long-term residual deficits of these patients. Initial assessment of COVID-19 survivors indicate that this disease could have multiple long-term effects leading to respiratory but also cardiovascular, renal or neurological sequelae $(9,10)$. Here, in the light on the complex immune dysregulation that occurs during acute infection $(11,12)$, we hypothesize that COVID-19 could have long-term impact on immune functions, such as the ones observed in patients that experienced bacterial sepsis (13). Indeed, immune profiling of SARS-CoV-2 recovered patients indicates persistent changes in the phenotype of innate (monocytes and granulocytes) but most importantly in adaptive (T, B, and NKT) immune cells (14-16). 
In this study, we explore the functional features of circulating monocytes in acute and convalescent COVID-19 patients. Stimulation with Toll-like receptor ligands associated with bacterial or viral patterns established the functional impairment of circulating mononuclear phagocytes in acutely ill and severely affected patients. This was not observed in convalescent patients but we observed a distinct pattern of cytokine production. We further evaluated the transcriptional and epigenomic profiles of $\mathrm{CD} 14^{+}$monocytes from patients that experienced severe COVID-19. Taken together, these data support an underlying epigenetic basis for functional reprograming of monocytes during and after severe COVID-19. 


\section{Results}

\section{Characteristics of the enrolled individuals}

To assess the eventual persistence of immune dysregulation, we enrolled patients during acute infection and convalescence phase (2 to 9 months after the onset of the symptoms, secondarily divided into "early recovery" for days 56 to 120 and "late recovery" for days 160 to 268 post symptoms onset). Patients were categorized according to the severity of the disease, "mild disease" represents patients who weren't hospitalized and "severe disease" corresponds to patients who required hospitalization due to COVID-19 (in regular ward or intensive care). Severe patients were more frequently male and older (Table 1$)$. Most patients $(89,7 \%)$ had a nasopharyngeal RT-PCR positive for SARS-CoV-2 at the hospital admission; otherwise, the diagnosis of COVID-19 was based on clinical and/or radiological features. During the acute phase, none of the mild patients died while death occurred in $38 \%$ of severe cases. Comorbidities and other demographic and disease features are shown in extended data (Table S.1).

Strong alteration of TLR-induced cytokine production by classical monocytes in severe patients during acute infection.

To evaluate the functionality of the innate immune cells in COVID-19, we performed ex vivo stimulation of whole blood with different pathogen-associated molecular patterns (PAMPs): bacterial LPS, a Toll-like receptor (TLR)4 ligand, R848, a synthetic ligand for TLR7/8 and Pam3CSK4, a synthetic ligand for TLR1/2. Medium alone served as unstimulated condition. We performed multiplex determination of cytokine levels in the supernatants and assessed cytokine production in monocytes at the single-cell level by multiparametric flow cytometry.

In acutely ill patients that did not require hospitalization, we observed few significant modulations of cytokine production in comparison to healthy controls: increased IFN $\beta$ levels in response to R848 and increased LPS-elicited IL10 and IL6 production. In sharp contrast, in 
severely ill patients, we observed strongly reduced levels of almost all cytokines in response to LPS or R848 stimulation with the exception of IFN $\beta$ which was significantly increased (Fig.1). Of note, in comparison to other stimuli, Pam3CSK4 induced low levels of some cytokines (CCL2, IL6, IL10) but to a similar extent in the different groups. Because whole blood is a complex mixture of cells that can directly or indirectly produce these cytokines in response to TLR stimulation, we also evaluated cytokine production at the single-cell level using flow cytometry. Consistent with our Luminex data, the proportion of CD1 $14^{\text {high }}$ monocytes expressing IL1 $\beta$, IL6, TNF $\alpha$ and IL12/23 in response to LPS or R848 was found to be decreased in severe patients in comparison to healthy subjects or mild disease patients. In contrast to these conditions, stimulation with Pam3CSK4 was weaker but was comparable in the 3 groups (Fig.2.A).

To fully apprehend the extent of this functional alteration, we were interested in evaluating the capacity of each cell to produce more than 1 cytokine simultaneously (i.e.polyfunctionality). The capacity to produce 3 or 4 cytokines concurrently was impaired in severe patients during acute COVID-19 in response to stimulation by LPS or R848 but not by Pam3CSK4 (Fig.2B). This latter parameter was increased in monocytes from mild COVID-19 patients as compared to controls. Taken together, these experiments demonstrate that the ability of monocytes to mount an appropriate pro-inflammatory response is impaired in severe COVID-19 patients upon stimulation through TLR4 or TLR7/8. When considering the most informative immune parameters in all acute COVID-19 patients, we observed a very strong correlation between the different responses to LPS and R848 as compared to the same analysis in naive subjects. This indicates that during SARS-CoV2 infection, modulation of the capacity of monocytes to produce different cytokines is highly coordinated and that the effect is global (Fig. S.1A).

Based on these data for our 24 severe patients, we defined whether these immune parameters were correlated with clinical outcome. As shown in Fig. 2C, a low degree of cytokine production was significantly associated with a higher risk of death among hospitalized 
patients indicating that innate immune paralysis is a key feature of the most severe forms of COVID-19.

Functional modulation of monocyte function in severe COVID-19 convalescent patients.

In order to define whether this immune dysfunction persists after the resolution of the acute infectious episode, we prospectively recruited patients 56 to 268 days after the onset of the symptoms. We quantified cytokine production in whole blood assays. Except for increased IFN $\beta$ concentrations in previously infected and hospitalized subjects, cytokine levels were found to be comparable in non-infected controls and severe convalescent patients, indicating that the global dysfunctional state observed during severe COVID-19 infection is not longlasting (Fig.3). Of note, although it did not reach statistical significance in the whole group, we also observed high basal CCL2 production in a subset of these patients. In convalescent patients from mild disease, there was a trend for higher IL1 $\beta$, IL6 and TNF production.

Interestingly, focusing on classical monocytes, patients that recovered from a severe disease showed an increased capability to produce IL1 $\beta$, IL6 and TNF $\alpha$ upon engagement of TLR1/2. On the other hand, in response to LPS stimulation, we noted a reduced proportion of IL12/23+ cells in the same group of patients (Fig.4A and B). This distinctive pattern appeared to concern mainly patients in the early phase of recovery (Fig.4C).

To visualize how all the different immune parameters globally vary across the clinical groups we used t-SNE, an analytical method that compares biological samples without taking into account sample classification (Fig.4D). We selected features that were significantly different between naïve controls, acute and convalescent patients (Fig.S.1B). This analysis shows an approximate 3-way separation with clusters enriched either for COVID-19 patients in the acute (cluster I) or convalescent stages (cluster III). Cluster I comprised a very clear subgroup of patients that were severely ill. In conclusion, alteration of TLR-responsiveness is a key feature of the most severe forms of COVID-19. Months after the recovery, the monocytes 
of these patients display a distinct pattern of cytokine production which suggests that the infectious event has induced a long-lasting functional reprograming.

\section{Monocytes from acute and convalescent COVID-19 patients display distinctive transcriptomic profiles}

To gain further insight into the molecular features of $\mathrm{CD} 14^{+}$monocytes in these severely affected COVID-19 patients during acute and convalescent stages we selected only severe patients from our cohort and age-and sex-matched controls and performed global transcriptional profiling. Characteristics of these individuals are shown in Table S.2. Since we observed differences in terms of cytokine production between early (56 to 120 post symptom onset) and late recovery stages (160 to 268 post symptoms onset) (Fig.4B), we analysed these groups separately. We observed a clear separation between samples from acute and healthy controls upon principal component analysis (Fig.5A). For samples from convalescent patients, those from early recovery stage formed a distinct cluster while those from late recovery stage were found to be embedded within the control group. In acute samples, we identified 339 statistically differentially expressed genes (DEG: 184 up- and 155 downregulated genes compared to controls, with a fold change $(F C)>2$ and a false discovery rate $(\mathrm{FdR})<0.05$, Fig.5B). Consistent with their altered functional response and recent reports $(17,18)$, we observed decreased expression of genes related to key immune pathways such as antigenic presentation, innate immune responses and MAPK and NF-KB signalling (JUNB, ATF3, NFkB2) in monocytes from patients suffering from severe COVID-19 (Fig.5B). We also observed increased expression of genes involved in key metabolic processes, including lipid metabolism (Fig.5C). Using the same criteria for samples from convalescent patients in the early recovery period, we identified 521 DEGs (318 up- and 203 downregulated genes compared to controls). Expression of multiple genes encoding chemokines was upregulated along with important intracellular immunomodulatory proteins and transcription factors (PPARG, FOSL1, MAFB, MAFF, ATF4, FOXO3). Hardly any DEGs were identified for the 
patients at the latter stage. Importantly, very few genes that were up or downregulated in the acute stage were found to be also modulated in the recovery groups (Fig.5B), indicating that the transcriptomic program induced by the acute infection is not persistent.

We also analyzed publicly available gene sets from scRNASeq data of acute COVID19 patients. In acute phase, we observed strong enrichment for genes identified in monocytes from the most severely affected patients (19). Schulte-Schrepping et al. described several blood monocyte subsets that arise in mild and severe patients (6). In monocytes from acute samples, we observed strong enrichment for marker genes of clusters $\mathrm{C} 2$ which corresponds to $\mathrm{HLA}-\mathrm{DR}{ }^{\text {lo }} \mathrm{CD} 163^{\text {hi }}$ cells that are the most abundant in early stages of the disease in severe patients. In monocytes from recovered patients, the strongest enrichment was seen for marker genes of cluster $\mathrm{C} 0$ (HLA-DR ${ }^{\circ} \mathrm{S} 100 \mathrm{~A}^{+}$cells) that dominates later in the course of the acute disease and of cluster C1 (HLA-DR ${ }^{\text {hiCD83 }}{ }^{+}$cells) that corresponds to activated cells. Monocytes from acute patients were also enriched for the MS1 gene signature derived from immature monocyte state in sepsis patients (20). In contrast, genes that were modulated in the recovery phase (i.e. several weeks after the sepsis) (21) were significantly up- or downregulated in early recovered patients, indicating potential common underlying mechanisms between these two clinical situations. Altogether, our transcriptomic data on CD14 ${ }^{+}$monocytes from acute severe COVID-19 patients indicate that these cells display an altered profile that could account for their decreased responsiveness to PAMPs. The distinctive transcriptional state of monocytes identified in recovery patients is probably not related to the same processes that occur in acutely ill patients and is not persistent in later stages of convalescence. This unique profile displays similarities with monocytes from patients that recovered from sepsis. 


\section{Monocytes from acute and convalescent severe COVID-19 patients display distinct profiles of chromatin accessibility}

To identify the epigenetic determinants of these distinct transcriptional and functional programs, we mapped chromatin accessibility by ATAC-Seq. As expected, we observed extensive modifications in monocytes from acute COVID-19 patients as 1617 and 1582 regions were found to be significantly more or less accessible in acute patients vs controls, respectively (Fig.6A). Strikingly, we also observed important changes in early but not late convalescing patients. A low number of differentially accessible regions (DARs) identified were common to both comparisons (Fig.6B). We used the Binding and Expression Target Analysis (BETA) package (22) to predict the activating or repressive function of these DARs. For acute patients, regulatory regions that were more or less accessible were clearly associated with genes that were up or downregulated at the transcriptional level, respectively (Fig.6C). For early convalescent patients, we also observed strong association between the regions that are more accessible and genes that are activated in this group. In contrast, the regions that were found to be less accessible were not clearly associated with down-regulated genes, suggesting that the most important regulatory features in this case are linked to gene activation rather than repression. These observations strongly suggest that epigenetic imprinting is responsible for the transcriptional signatures identified in acute and early convalescing patients. For example, in acute patients, less accessible regions were found in the loci of $I L 1 B$ or IL1R1 genes (Fig. 6D). In early convalescent patients, we observed increased accessibility at the loci of the same genes.

Next, we performed gene-ontology analysis using Genomic Regions Enrichment of Annotations Tool (GREAT) (23). Consistent with our transcriptomic data, we observed that regulatory regions that are less accessible in monocytes from acute patients were associated with genes involved in inflammatory response, lipid metabolism or cytokine-mediated signalling pathways (Fig 6E). We observed a mirror image in recovery patients with increased accessibility associated to genes involved in innate immune response and TLR signalling 
pathway but also in wound healing. We then scanned for binding motifs at the center of ATAC peaks located in these sets of regions. Analysis of putative TF site enrichment in patients vs control-specific regions indicated a strong and significant enrichment for distinct motifs in both groups (Fig.7A). Consistent with the decreased expression of NFKB2 and JUNB (Fig 7B), we observed an overrepresentation for AP1 (Jun/Fos), REL binding motifs in regions that were less accessible in monocytes from acute patients. We also identified IRF1 and STAT motifs in these regions, suggesting that multiple inflammatory modules are deactivated at this stage of the disease. In sharp contrast, in regions that were more accessible in convalescent patients, we observed a strong enrichment for AP-1 and MARE-containing motifs (Fig.7A). We identified both motifs in a substantial proportion of these regions (845/3458 regions, linked to 121 significantly up-regulated genes in recovery patients in comparison to controls, termed AP1/MAF transcriptional module) For example, such motifs were identified in the locus of the PPARG gene (Fig.7C). Of note, we observed increased levels of MAFB, MAFF but also FOSL1, a partner of Jun family members that displays an important role in orchestrating the expression of genes related to wound response, Toll-like receptor activation, and interleukin signaling of macrophages (24). Given that JUN proteins also form heterodimers with members of the MAF family (25), it is tempting to speculate that upregulation of MAFB expression in these cells redirects AP-1 complexes to these genomic targets thereby promoting local chromatin remodeling. Consistent with this hypothesis, we observed a strong correlation between MAFB and FOSL1 levels and expression of the AP1/MAF transcriptional module in the whole cohort (Fig.7D). These correlations were less obvious with other members of the MAF, JUN, FOS or BATF families. Finally, we observed strong enrichment for MAF-responsive genes in monocytes from convalescent patients in comparison to controls (Fig.7E) (26). MafBdependent genes rather than cMAF-dependent genes were enriched, reinforcing the potential contribution of this specific transcription factor. Taken together, these data support the notion that the transcriptional program and functional properties of monocytes in acute or convalescing severe COVID-19 patients have strong epigenetic determinants. 


\section{Discussion}

Multiple reports now indicate that COVID-19 infection is associated with drastic changes in the myeloid compartment, particularly in patients with a severe course of disease (18,27-29). It has been shown that severe and fatal COVID-19 leads to accumulation of HLA-DR ${ }^{\text {lo }}$ monocytes with potentially suppressive and dysfunctional features. Here, to evaluate the function of these cells more thoroughly, we first assessed the capacity of circulating cells from mild and severe patients to produce cytokines in response to prototypical TLR ligands. We observed that the ability of monocytes from severe COVID-19 patients to produce inflammatory cytokines was severely impaired. Of note, stimulation with the TLR1/2 ligand Pam3CSK4 was generally less affected than with other ligands and we observed even higher levels of IFN $\beta$ in LPS- or R848-stimulated whole blood cultures, indicating complex functional changes. Moreover, among severely ill patients, decreased ability of monocytes to produce several cytokines simultaneously was associated with a higher risk of mortality. Our transcriptomic and epigenomic analysis of $\mathrm{CD} 14^{+}$monocytes indicated that the functional alterations identified in acute severe patients are accompanied by decreased basal activity of key modules involved in TLR signalling pathways, including NF-kB and AP-1. Multiple molecular mechanisms may account for the unique phenotypic and functional features of monocytes in this context. The intense systemic inflammation could have direct effects on circulating cells but also trigger emergency hematopoiesis and the release of immature cells from the bone marrow as observed in sepsis (30). In addition, treatment itself could also have an impact on the functional features on circulating monocytes as all hospitalized patients received corticosteroids as standard of care and sometimes other immunomodulatory drugs (including hydroxychloroquine, anti-interleukin therapies and antiviral drugs).

Next, to define whether this hematopoietic reprograming during acute severe COVID19 infection could have long-term impact on monocyte function, we recruited patients that had recovered from the disease. The production of cytokines in patients convalescing from mild or severe disease was globally comparable to that of SARS-CoV2 naive controls. Yet, at the 
single-cell level, we observed subtle changes including decreased production of IL-12 in response to LPS and increased responsiveness to TLR1/2 ligation in patients that recovered from severe disease. This was more obvious in the first few months after the disease, suggesting that the impact of COVID-19 infection/hospitalization on monocyte function fades after a longer period. In these early convalescent patients (2-3 months post-infection), we observed striking molecular profiles characterized by epigenomic reprogramming reminiscent of trained immunity. This term has been proposed for the persistent enhanced state of the innate immune response following exposure to certain infectious agents or vaccines, which may result in increased resistance to related or unrelated pathogens (31). However, similar processes may also result in hypo-responsiveness and be deleterious, for example in the context of chronic metabolic and inflammatory diseases such as liver cirrhosis - a condition associated with increased susceptibility to infections (32). More recently, Wimmers et al showed that administration of seasonal influenza vaccine induced persistent epigenomic changes in myeloid cells leading to innate refractoriness associated with decreased AP-1 activity (33). Multiple mechanisms may account for these long-lasting effects on innate immune cells. In the context of BCG immunization, exposure to LPS or sepsis, it involves modulation of myeloid progenitors in the bone marrow (34-36). In the context of COVID-19, they could also be the consequence of persistent viral antigen expression (detected up to 4 months after the onset of the disease) (37) or result from the modulation of the adaptive compartment as the majority of SARS-CoV2 specific CD8 T cells acquire a terminally differentiated phenotype (38).

Single-cell epigenomic profiling identified distinct states among classical monocytes and indicated that "activated" or "trained" subsets were enriched in convalescing COVID-19 patients (39). In line with this report, we observed that the epigenomic state of monocytes from patients that recovered from severe COVID-19 was associated with heightened AP-1 and MAF activities. We uncovered the potential contribution of FOSL1 and MafB in this functional reprograming. FOSL1 has been shown to regulate pro- and anti-inflammatory cytokine 
expression in macrophages, modulating profibrotic responses (40) and promoting lung or joint inflammation $(41,42)$. MafB is also a key regulator involved in functional programing of macrophages in the context of tissue imprinting (43), lipid/cholesterol-rich environments or wound healing (44). Of note, the balance between MAF and MafB in alveolar macrophages could shape the response to SARS-CoV2 (45).

This functional reprograming of monocytes in convalescing COVID-19 patients could have both beneficial and detrimental consequences. In particular, an emerging complication of COVID-19 infection is a prolonged period of lingering symptoms post-infection (10). An hyperinflammatory state seems to be a cardinal feature of this syndrome that is also associated with increased risk for sudden cardiovascular events (46). Hence, the epigenomic changes in circulating monocytes we describe here could participate to cytokine-driven endothelial dysfunction (47).

We are mindful of the limitations of the present study. It would be important to follow these patients longitudinally as they represent a very heterogeneous population. Hence, it is possible that more subtle variations, for example in mild patients, have been overlooked. In addition, the differences we identified here using bulk approaches probably reflect the changes in the most abundant subsets of monocytes. Furthermore, we cannot exclude a selection bias in convalescent patients that survived from an acute infection. Finally, multiple factors that could influence the function of these cells were not taken into account, including treatment modalities and co-morbidities.

In conclusion, it is now clear that immune responses are strongly influenced by past and present interactions of the host immune system with its environment (48). We showed here that severe COVID-19 infection has a profound impact on the differentiation status and function of circulating monocytes both during the acute and the convalescent phases in a completely distinct manner. This could have important implications for our understanding of short and long-term COVID19-related morbidity and mortality. 


\section{Material and Methods}

\section{Patient recruitment}

For this study, between June 2020 and January 2021, we recruited 97 patients during acute infection or at early and late recovery phases. Patients were categorized according to the severity of the disease, "mild disease" represents patients who weren't hospitalized and "severe disease" corresponds to patients who were hospitalized due to COVID-19 (in regular ward or intensive care). Among acute infections, 11 were mild and 24 severe. 19 patients recovered from mild and 43 from severe disease. As controls, we recruited 32 SARS-CoV2naïve individuals (negative nasopharyngeal RT-PCR and serology for SARS-CoV-2) among health-care workers and nursing home residents (49) that were age- and sex-matched to severe COVID-19 patients.

The protocol was approved by the local ethics committee (Epicura, Baudour, Belgium: P2020011; Erasme, Brussels, Belgium: B4062020000029; CHU Saint-Pierre, Brussels, Belgium: CE200910) and was conducted according to the guidelines of the 1975 Declaration of Helsinki. Written informed consent was obtained from all patients or their designated family members.

\section{Blood collection}

All blood draws were performed in the hospital by a trained phlebotomist. Peripheral blood was drawn via sterile venipuncture into sodium-heparin vacutainers. Blood samples were kept at room temperature (RT) and processed within 4 hours of the blood draw. 
PRR stimulation

To minimize technical artifacts, we used a highly standardized, stringently controlled protocol as described previously (50). Briefly, premade 96-well plates contained the following specific PRR ligands were prepared: PAM3CSK4 (PAM; TLR2/1; InvivoGen) at $1 \mu \mathrm{g} / \mathrm{ml}$; LPS (TLR4; InvivoGen) at $10 \mathrm{ng} / \mathrm{ml}$; R848 (TLR7/8; InvivoGen) at $10 \mu \mathrm{M}$ and media alone. Whole blood, diluted 1:1 with sterile prewarmed RPMI 1640, was added to each well containing the specific TLR ligands.

For the intracellular cytokine staining (ICS), $10 \mu \mathrm{g} / \mathrm{ml}$ of brefeldin A (final concentration -SigmaAldrich) was added to each well, and samples were incubated for $6 \mathrm{~h}$ at $37^{\circ} \mathrm{C}$ in $5 \% \mathrm{CO}$, then treated with $2 \mathrm{mM}$ EDTA (final concentration) for $10 \mathrm{~min}$ at $37^{\circ} \mathrm{C}$. The cells were collected and resuspended in BD FACS Lysing Solution, placed into fresh tubes, and stored at $-80^{\circ} \mathrm{C}$.

For multiplex analysis, samples were incubated with whole blood for $24 \mathrm{~h}$ at $37^{\circ} \mathrm{C}$ in $5 \% \mathrm{CO}$. The supernatant was collected and stored at $-80^{\circ} \mathrm{C}$.

Intracellular cytokine staining and flow cytometric acquisition

Frozen tubes were thawed and spun, pellets were washed multiple times with wash buffer (PBS, 5\% FCS). Cells were stained for 30 min at RT with the following antibodies: CD45, CD3, CD19, CD66, HLA-DR, CD14, CD11C (BD Biosciences), CD16 (Biolegend), CD123 (eBioscience). Samples were washed again using wash buffer and then incubated for 20 min at $4^{\circ}$ with BD CytofixCytoperm solution, before being stained for $20 \mathrm{~min}$ at RT with the following intracytoplasmic antibodies: TNF $\alpha$, IL1 $\beta$ (eBioscience), IL12/23p40, IL6 (Invitrogen). Flow cytometric data acquisition was performed on a Cytoflex LX Beckmann \& Coulter and analyzed using FlowJo software (Fig.S.3). 
Cytokine measurement in whole blood culture supernatant

Multiplex assays from Bio-techne were used to measure the cytokine (TNF $\alpha$, IL6, IL12p70, IL1 $\beta$, IL10, IFNa, IFN $\beta$, IFN $\gamma$, ) and chemokine (CXCL10, CCL2) levels in the supernatants.

Isolation of peripheral blood mononuclear cells (PBMCs) and sorting of CD14+ monocytes

PBMCs were isolated from sodium-heparinised whole blood by density gradient centrifugation using Lymphoprep ${ }^{\mathrm{TM}}$ (ProteoGenix) and Leuco-Sep tubes (Greiner) and PBMCs were frozen in FCS with 10\% DMSO in liquid nitrogen. PBMCs were thawed, stained with trypan blue and counted to ensure a majority of live cells. CD14+ monocytes were isolated by fluorescenceactivated cells sorting (FACS) on a SONY SH800S Cell Sorter (Fig.S.4). Post- sort purity was higher than $90 \%$.

RNA sequencing

15.000 to 200.000 CD $14^{\text {hi }}$ monocytes from 11 healthy controls, 13 acute and 20 convalescent patients were isolated by FACS directly in TRIzol reagent (ThermoFisher scientific). After chloroform extraction, RNA isolation was performed using a RNeasy kit (Qiagen) and sample quality was tested on a Fragment Analyzer (Agilent). Indexed cDNA libraries were obtained using the Ovation Solo RNA-Seq System (Tecan) following manufacturer's recommendations. The multiplexed libraries were loaded on a NovaSeq 6000 (Illumina) using a S2 flow cell and $25 \times 10^{6}$ paired-end reads/sample were produced using a 200 cycle Kit. The RNA sequencing was performed by BRIGHTcore ULB-VUB, Belgium (http://www.brightcore.be). Adapters were removed with Trimmomatic-0.36 with the following parameters: Truseq3-PE.fa:2:30:10 LEADING:3 TRAILING:3 SLIDINGWINDOW:4:15 MINLEN:36 HEADCROP:4. Reads were then mapped to the reference genome GRCh38 by using STAR_2.5.3a software with default parameters. We then sorted the reads from the alignment according to chromosome positions 
and indexed the resulting BAM-files. Read counts in the alignment BAM-files that overlap with the gene features were obtained using HTSeq-0.9.1 with "--nonunique all" option (if the read pair aligns to more than one location in the reference genome, it is counted in all features to which it was assigned and scored multiple times). Genes with no raw read count greater or equal to 20 in at least 1 sample were filtered out with an $R$ script, raw read counts were normalized and a differential expression analysis was performed with DESeq2 by applying an adjusted $p$-value $<0.05$ and an absolute log2-ratio larger than 1.

ATAC

Assay for transposase accessible chromatin (ATAC) followed by sequencing was performed as follows: 10.000 to 50.000 sorted $\mathrm{CD} 14^{+}$monocytes from 11 healthy controls, 14 acute and 21 convalescent patients were collected in $1 \mathrm{~mL}$ of cell culture media at $4^{\circ} \mathrm{C}$. Cells were centrifuged, then cell pellets were resuspended in $50 \mu \mathrm{L}$ of lysis buffer (Tris $\mathrm{HCl} 10 \mathrm{mM}, \mathrm{NaCl}$ $10 \mathrm{mM}, \mathrm{MgCl} 23 \mathrm{mM}$, Igepal 0,1\%) and centrifuged $(500 \mathrm{~g})$ for $25 \mathrm{~min}$ at $4^{\circ} \mathrm{C}$. Supernatant was discarded and nuclei were resuspended in $50 \mu \mathrm{L}$ of reaction buffer $(\mathrm{Tn} 5$ transposase $2,5 \mu \mathrm{L}$, TD buffer $22,5 \mu \mathrm{L}$ and $25 \mu \mathrm{L} \mathrm{H} 2 \mathrm{O}$ - Nextera DNA sample preparation kit, Illumina). The reaction was performed for 30 min at $37^{\circ} \mathrm{C}$. DNA was purified using the MinElute purification kit (QIAGEN). Purified DNA was amplified and indexed by PCR using NEBNext High-Fidelity 2× PCR Master Mix (New England Biolabs) with 10-12 cycles. Amplified libraries were purified using MinElute PCR Purification Kit (Qiagen), followed by a double AMPURE XP purification (0,5:1 and 1.2:1 ratios) and quality controlled using a Fragment Analyzer High-Sensitivity DNA Analysis kit (Agilent). Paired-end sequencing was performed on NovaSeq platform (Illumina).

Adapters in obtained reads were removed with Trimmomatic 0.36 with the following parameters: Nextera1.fa:1:25:6 LEADING:3 TRAILING:3 SLIDINGWINDOW:4:15 MINLEN:36. Paired-end reads were mapped to human genome GRCh38 with Bowtie2 $(51,52)$ using the following parameters for paired-end reads -X $2000-\mathrm{fr}$-very-sensitive -no- 
discordant -no-mixed -non-deterministic. Reads from the alignment were sorted and indexed according to chromosomes. Reads located within the blacklist of the ENCODE projectDuplicate reads were removed with MarkDuplicates tools (Picard suite). Peaks were called with MACS2 (53) using the following parameters: -f BAMPE -g mm -q 0.05 --nomodel -call-summits -B -SPMR.

Regions obtained by MACS2 were merged to create an atlas containing all obtained peaks for all the populations using bedtools (54) with a minimum overlapping of $1 \mathrm{bp}$. Merged regions were subject to differential analysis using csaw workflow (55) .For downstream visualisation, a scaling factor was calculated using deepTools package (56) to normalise peak intensity to FRiP (fraction of reads in peaks) and generate bigwig files.

For Gene ontology analysis, we introduced BED files from differential ATAC-seq peaks to GREAT tool with default parameters (23). For motif analysis, Ciiider algorithm (http://ciiider.com/) was used to perform motif enrichment in the differentially accessible regions.

We used BETA package (22) with default parameters to integrate ATAC-seq (differentially accessible regions) and RNA-seq (transcriptome) data and evaluate the regulatory potential of chromatin accessibility to promote/repress genes expression.

Data availability

RNA-seq and ATAC-seq data that support the findings reported in this study have been deposited in the GEO Repository with the accession code (pending).

\section{Unsupervised analysis}

First, the centered and scaled cellular phenotypic data corresponding to patients in the different clinical categories was visualized in two dimensions using t-distributed stochastic 
neighbor embedding (t-SNE). The observed clustering was stable across t-SNE technical replicates. To assess which cellular phenotypes, i.e. features, best explained the differences across the disease stages, we computed P-values from a Kruskal-Wallis test.

\section{Statistical analysis}

Group comparisons were performed using two-way ANOVA followed by Bonferroni posttests or Kruskal-Wallis test with Dunn's correction for multiple testing when appropriate. Statistical analysis was conducted using R, Prism Version 5 (GraphPad, La Jolla, CA) or IBM SPSS Statistics 22. The level of statistical significance was set at $p$-value $<0.05$.

\section{Author Contributions}

EB conducted most of the experiments. EB, VDM, PP and AM elaborated the clinical studies. $\mathrm{ND}, \mathrm{DG}, \mathrm{COC}$ and SS contributed to the recruitment of the patients and the controls. AA, VO, MN, ST, IVD and FL contributed to some experiments. AA performed bioinformatics analysis. EB and AA analyzed the data and prepared the figures. JD and HX provided input for data analysis and interpretation. EB and SG wrote the manuscript. SG supervised the work. All authors were involved in critically revising the manuscript for important intellectual content. All authors had full access to the data and approved the manuscript before it was submitted by the corresponding author.

\section{Acknowledgments}

The authors wish to thank Laure Vandenheede, Catherine Quoidbach, Sophia Mora and Sina Karimi for their availability and kindness during the enrolment of convalescents patients ; Sébastien Denanglaire for his invaluable help and advises regarding cytometry and Maria Goossens and Isabelle Desombere for their work in the PICOV study. This study was 
supported by the Fonds National de la Recherche Scientifique (FRS-FNRS, Belgium), the European Regional Development Fund (ERDF) of the Walloon Region (Wallonia-Biomed portfolio, 411132-957270), the Fonds ERASME (Erasme Hospital, Université Libre de Bruxelles). EB is a research fellow, ND a post-doctoral fellow, SG is a senior research associate and AM a research director of the FRS-FNRS.

Conflict of interest : The authors have declared that no conflict of interest exists 


\section{Bibliography}

1. Teuwen L-A, Geldhof V, Pasut A, Carmeliet P. COVID-19: the vasculature unleashed. Nature reviews Immunology. 2020;20(7):389-91.

2. Perico L, Benigni A, Casiraghi F, Ng LFP, Renia L, Remuzzi G. Immunity, endothelial injury and complement-induced coagulopathy in COVID-19. Vol. 17, Nature Reviews Nephrology. Nature Research; 2021. p. 46-64.

3. Giamarellos-Bourboulis EJ, Netea MG, Rovina N, Akinosoglou K, Antoniadou A, Antonakos N, et al. Complex Immune Dysregulation in COVID-19 Patients with Severe Respiratory Failure. Cell host \& microbe. 2020;27(6):992-1000.e3.

4. Zhang D, Guo R, Lei L, Liu H, Wang Y, Wang Y, et al. Frontline Science: COVID-19 infection induces readily detectable morphologic and inflammation-related phenotypic changes in peripheral blood monocytes. Journal of Leukocyte Biology. 2021 Jan $11 ; 109(1)$.

5. Knoll R, Schultze JL, Schulte-Schrepping J. Monocytes and Macrophages in COVID19. Frontiers in Immunology [Internet]. 2021 Jul 21;12. Available from: https://www.frontiersin.org/articles/10.3389/fimmu.2021.720109/full

6. Schulte-Schrepping J, Reusch N, Paclik D, Baßler K, Schlickeiser S, Zhang B, et al. Severe COVID-19 Is Marked by a Dysregulated Myeloid Cell Compartment. Cell. 2020 Sep 17;182(6):1419-1440.e23.

7. Arunachalam PS, Wimmers F, Mok CKP, Perera RAPM, Scott M, Hagan T, et al. Systems biological assessment of immunity to mild versus severe COVID-19 infection in humans. Science. 2020;369(6508):1210-20.

8. Hadjadj J, Yatim N, Barnabei L, Corneau A, Boussier J, Smith N, et al. Impaired type I interferon activity and inflammatory responses in severe COVID-19 patients. Science. 2020 Aug 7;369(6504):718-24.

9. Wang F, Kream RM, Stefano GB. Long-term respiratory and neurological sequelae of COVID-19. Medical Science Monitor. 2020 Nov 1;26.

10. Nalbandian A, Sehgal K, Gupta A, Madhavan M v., McGroder C, Stevens JS, et al. Post-acute COVID-19 syndrome. Vol. 27, Nature Medicine. Nature Research; 2021. p. 601-15.

11. Giamarellos-Bourboulis EJ, Netea MG, Rovina N, Akinosoglou K, Antoniadou A, Antonakos N, et al. Complex Immune Dysregulation in COVID-19 Patients with Severe Respiratory Failure. Cell Host \& Microbe. 2020 Jun;27(6):992-1000.e3.

12. Yao C, Bora SA, Parimon T, Zaman T, Friedman OA, Palatinus JA, et al. Cell-TypeSpecific Immune Dysregulation in Severely III COVID-19 Patients. Cell Reports. 2021 Jan 5;34(1).

13. Venet F, Monneret $\mathrm{G}$. Advances in the understanding and treatment of sepsis-induced immunosuppression. Nature Reviews Nephrology. 2018;14(2):121-37.

14. Orologas-Stavrou N, Politou M, Rousakis P, Kostopoulos I V., Ntanasis-Stathopoulos I, Jahaj E, et al. Peripheral Blood Immune Profiling of Convalescent Plasma Donors Reveals Alterations in Specific Immune Subpopulations Even at 2 Months Post SARSCoV-2 Infection. Viruses. 2020;13(1):1-16. 
15. Files JK, Boppana S, Perez MD, Sarkar S, Lowman KE, Qin K, et al. Sustained cellular immune dysregulation in individuals recovering from SARS-CoV-2 infection. Journal of Clinical Investigation. 2021 Jan 4;131(1).

16. Benhnia R-E-IM. Dendritic cell deficiencies persist seven months after SARS-CoV-2 infection. Available from: https://doi.org/10.1101/2021.03.18.436001

17. Liu C, Martins AJ, Lau WW, Rachmaninoff N, Chen J, Imberti L, et al. Time-resolved systems immunology reveals a late juncture linked to fatal COVID-19. Cell. $2021 \mathrm{Apr}$ 1;184(7):1836-1857.e22.

18. Wilk AJ, Rustagi A, Zhao NQ, Roque J, Martínez-Colón GJ, McKechnie JL, et al. A single-cell atlas of the peripheral immune response in patients with severe COVID-19. Nature Medicine. 2020 Jul 1;26(7):1070-6.

19. Wilk AJ, Lee MJ, Wei B, Parks B, Pi R, Ranganath T, et al. Multi-omic profiling reveals widespread dysregulation of innate immunity and hematopoiesis in COVID-19. Available from: https://doi.org/10.1101/2020.12.18.423363

20. Reyes M, Filbin MR, Bhattacharyya RP, Billman K, Eisenhaure T, Hung DT, et al. An immune-cell signature of bacterial sepsis. Vol. 26, Nature Medicine. Nature Research; 2020. p. 333-40.

21. Shalova IN, Lim JY, Chittezhath M, Zinkernagel AS, Beasley F, Hernández-Jiménez E, et al. Human monocytes undergo functional re-programming during sepsis mediated by hypoxia-inducible factor-1 $\alpha$. Immunity. 2015 Mar 17;42(3):484-98.

22. Wang S, Sun $\mathrm{H}$, Ma J, Zang C, Wang C, Wang J, et al. Target analysis by integration of transcriptome and ChIP-seq data with BETA. Nature Protocols. 2013 Dec 21;8(12).

23. McLean CY, Bristor D, Hiller M, Clarke SL, Schaar BT, Lowe CB, et al. GREAT improves functional interpretation of cis-regulatory regions. Nature Biotechnology. 2010 May 2;28(5).

24. Hannemann N, Cao S, Eriksson D, Schnelzer A, Jordan J, Eberhardt M, et al. Transcription factor Fra-1 targets arginase-1 to enhance macrophage-mediated inflammation in arthritis. Journal of Clinical Investigation. 2019 May 28;129(7).

25. Bejjani F, Evanno E, Zibara K, Piechaczyk M, Jariel-Encontre I. The AP-1 transcriptional complex: Local switch or remote command? Biochimica et Biophysica Acta (BBA) - Reviews on Cancer. 2019 Aug;1872(1).

26. Kang K, Park SH, Chen J, Qiao Y, Giannopoulou E, Berg K, et al. Interferon-Y Represses M2 Gene Expression in Human Macrophages by Disassembling Enhancers Bound by the Transcription Factor MAF. Immunity. 2017 Aug;47(2).

27. Zhou R, To KKW, Wong YC, Liu L, Zhou B, Li X, et al. Acute SARS-CoV-2 Infection Impairs Dendritic Cell and T Cell Responses. Immunity. 2020 Oct 13;53(4):864-877.e5.

28. Bost $P$, de Sanctis $F$, Canè $S$, Ugel $S$, Donadello $K$, Castellucci $M$, et al. Deciphering the state of immune silence in fatal COVID-19 patients. Nature Communications. 2021 Dec 5;12(1).

29. Parackova Z, Zentsova I, Bloomfield M, Vrabcova P, Smetanova J, Klocperk A, et al. Disharmonic Inflammatory Signatures in COVID-19: Augmented Neutrophils' but Impaired Monocytes' and Dendritic Cells' Responsiveness. Cells. 2020 Sep 29;9(10). 
30. Reyes M, Filbin MR, Bhattacharyya RP, Sonny A, Mehta A, Billman K, et al. Title: Induction of a regulatory myeloid program in bacterial sepsis and severe COVID-19. Available from: https://doi.org/10.1101/2020.09.02.280180

31. Netea MG, Joosten LAB, Latz E, Mills KHG, Natoli G, Stunnenberg HG, et al. Trained immunity: A program of innate immune memory in health and disease. Science. 2016 Apr 22;352(6284).

32. Weichselbaum L, Azouz A, Smolen KK, Das J, Splittgerber M, Lepida A, et al. Epigenetic basis for monocyte dysfunction in patients with severe alcoholic hepatitis. Journal of Hepatology. 2020 Aug 1;73(2):303-14.

33. Wimmers F, Donato M, Kuo A, Ashuach T, Gupta S, Li C, et al. The single-cell epigenomic and transcriptional landscape of immunity to influenza vaccination. Cell [Internet]. $2021 \quad$ Jul;184(15):3915-3935.e21. Available from: https://linkinghub.elsevier.com/retrieve/pii/S0092867421006929

34. Davis FM, Schaller MA, Dendekker A, Joshi AD, Kimball AS, Evanoff H, et al. Sepsis Induces Prolonged Epigenetic Modifications in Bone Marrow and Peripheral Macrophages Impairing Inflammation and Wound Healing. Arteriosclerosis, thrombosis, and vascular biology. 2019;39(11):2353-66.

35. Mitroulis I, Ruppova K, Wang B, Chen L-S, Grzybek M, Grinenko T, et al. Modulation of Myelopoiesis Progenitors Is an Integral Component of Trained Immunity. Cell. 2018 Jan;172(1-2):147-161.e12.

36. de Laval B, Maurizio J, Kandalla PK, Brisou G, Simonnet L, Huber C, et al. C/EBP $\beta$ Dependent Epigenetic Memory Induces Trained Immunity in Hematopoietic Stem Cells. Cell Stem Cell. 2020 May;26(5).

37. Gaebler C, Wang Z, Lorenzi JCC, Muecksch F, Finkin S, Tokuyama M, et al. Evolution of antibody immunity to SARS-CoV-2. Nature. 2021 Mar 25;591(7851).

38. Dan JM, Mateus J, Kato Y, Hastie KM, Yu ED, Faliti CE, et al. Immunological memory to SARS-CoV-2 assessed for up to 8 months after infection. Science. 2021 Feb $5 ; 371(6529)$.

39. You M, Chen L, Zhang D, Zhao P, Chen Z, Qin EQ, et al. Single-cell epigenomic landscape of peripheral immune cells reveals establishment of trained immunity in individuals convalescing from COVID-19. Nature Cell Biology. 2021 Jun 1;23(6):62030.

40. Rajasekaran S, Reddy NM, Zhang W, Reddy SP. Expression profiling of genes regulated by Fra-1/AP-1 transcription factor during bleomycin-induced pulmonary fibrosis. BMC Genomics. 2013;14(1).

41. Hannemann N, Cao S, Eriksson D, Schnelzer A, Jordan J, Eberhardt M, et al. Transcription factor Fra-1 targets arginase-1 to enhance macrophage-mediated inflammation in arthritis. Journal of Clinical Investigation. 2019 May 28;129(7).

42. Vaz M, Reddy NM, Rajasekaran S, Reddy SP. Genetic Disruption of Fra-1 Decreases Susceptibility to Endotoxin-Induced Acute Lung Injury and Mortality in Mice. American Journal of Respiratory Cell and Molecular Biology. 2012 Jan;46(1). 
43. Lavin Y, Winter D, Blecher-Gonen R, David E, Keren-Shaul H, Merad M, et al. TissueResident Macrophage Enhancer Landscapes Are Shaped by the Local Microenvironment. Cell. 2014 Dec;159(6).

44. Hamada M, Tsunakawa $\mathrm{Y}$, Jeon H, Yadav MK, Takahashi S. Role of MafB in macrophages. Experimental Animals. 2020;69(1).

45. Vega MA, Simón-Fuentes M, González de la Aleja A, Nieto C, Colmenares M, Herrero $\mathrm{C}$, et al. MAFB and MAF Transcription Factors as Macrophage Checkpoints for COVID19 Severity. Frontiers in Immunology. 2020 Nov 18;11.

46. Patell $R$, Bogue $T$, Koshy $A$, Bindal $P$, Merrill $M$, Aird WC, et al. Postdischarge thrombosis and hemorrhage in patients with COVID-19. Blood. 2020 Sep 10;136(11).

47. Chioh FW, Fong S-W, Young BE, Wu K-X, Siau A, Krishnan S, et al. Convalescent COVID-19 patients are susceptible to endothelial dysfunction due to persistent immune activation. eLife. 2021 Mar 23;10.

48. Muraille E, Goriely S. The nonspecific face of adaptive immunity. Current Opinion in Immunology. 2017;48.

49. Goossens ME, Neven KY, Pannus P, Barbezange C, Thomas I, van Gucht S, et al. Outlining the Prior Infection with SARS-CoV-2 study (PICOV)-preliminary findings on symptoms in nursing home residents and staff.

50. Smolen KK, Cai B, Gelinas L, Fortuno ES, Larsen M, Speert DP, et al. Single-Cell Analysis of Innate Cytokine Responses to Pattern Recognition Receptor Stimulation in Children across Four Continents. The Journal of Immunology. 2014 Sep 15;193(6).

51. Langmead B, Trapnell C, Pop M, Salzberg SL. Ultrafast and memory-efficient alignment of short DNA sequences to the human genome. Genome Biology. 2009;10(3).

52. Langmead B, Salzberg SL. Fast gapped-read alignment with Bowtie 2. Nature Methods. 2012 Apr 4;9(4).

53. Zhang Y, Liu T, Meyer CA, Eeckhoute J, Johnson DS, Bernstein BE, et al. Model-based Analysis of ChIP-Seq (MACS). Genome Biology. 2008;9(9).

54. Quinlan AR, Hall IM. BEDTools: a flexible suite of utilities for comparing genomic features. Bioinformatics. 2010 Mar 15;26(6).

55. Reske JJ, Wilson MR, Chandler RL. ATAC-seq normalization method can significantly affect differential accessibility analysis and interpretation. Epigenetics \& Chromatin. 2020 Dec 22;13(1).

56. Ramírez F, Dündar F, Diehl S, Grüning BA, Manke T. deepTools: a flexible platform for exploring deep-sequencing data. Nucleic Acids Research. 2014 Jul 1;42(W1). 
Mild $(n=11) \quad$ Severe $(n=24) \quad$ Mild $(n=19) \quad$ Severe $(n=43)$
Patients characteristics

Age

Sex

Disease features

ICU

Outcome

Time between illness onset and sampling

\begin{tabular}{|c|c|c|c|c|c|c|}
\hline & Mild $(n=11)$ & Severe $(n=24)$ & Mild $(n=19)$ & Severe $(n=43)$ & & \\
\hline Female & $\begin{array}{r}37(16-55) \\
7(64 \%)\end{array}$ & $\begin{array}{r}60(26-81) \\
9(38 \%)\end{array}$ & $\begin{array}{r}46(23-70) \\
9(47 \%)\end{array}$ & $\begin{array}{r}53(29-73) \\
15(35 \%)\end{array}$ & $\begin{array}{r}64(25-91) \\
17(53 \%)\end{array}$ & $\begin{array}{r}0,000^{*} \\
0,330\end{array}$ \\
\hline \multirow{2}{*}{ Death } & $0(0 \%)$ & $\begin{array}{r}17(71 \%) \\
9(38 \%)\end{array}$ & $0(0 \%)$ & $\begin{array}{r}18(42 \%) \\
0(0 \%)\end{array}$ & - & $\begin{array}{l}0,000^{*} \\
0,000^{*}\end{array}$ \\
\hline & $8(7-10)$ & $11(1-19)$ & $150(65-199)$ & $135(56-268)$ & - & \\
\hline
\end{tabular}

Table.1 : Demographics and clinical data. Data are presented as mean (min-max) or number of patients (\%). Continuous variables were compared by Kruskal-Wallis test, followed by Bonferroni posttests. Dichotomous variables were analysed with Chi-square test.

ICU, intensive care unit 


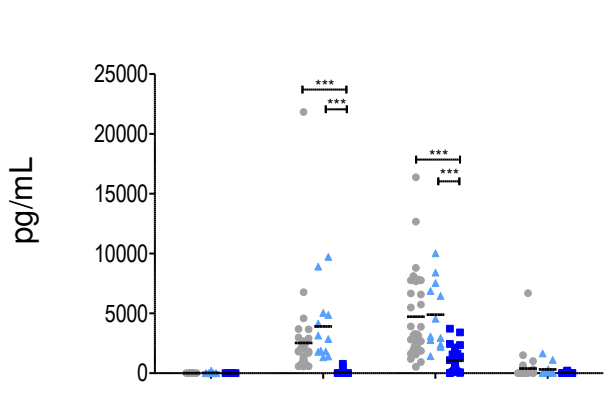

IL6

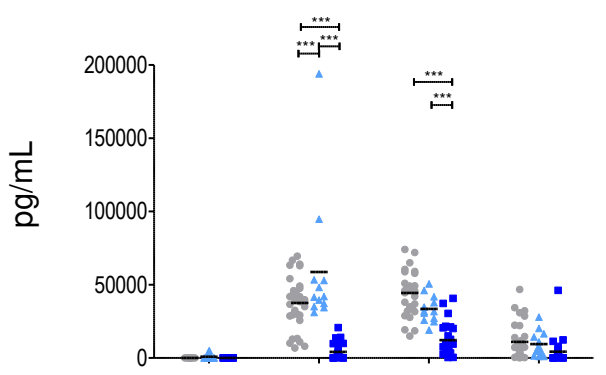

TNFa

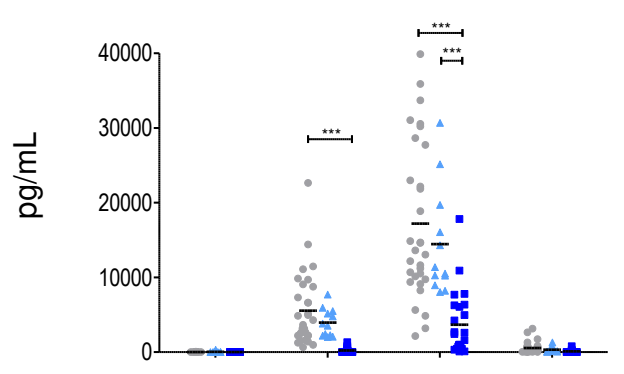

IL12p70
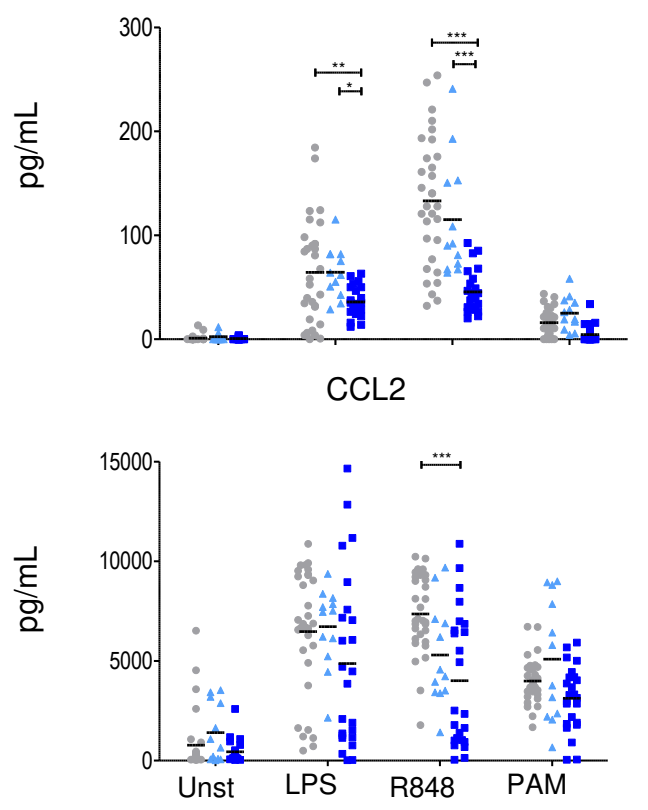

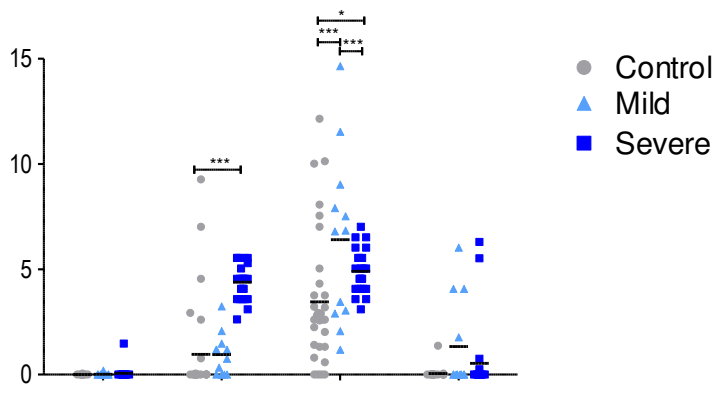

IFNa

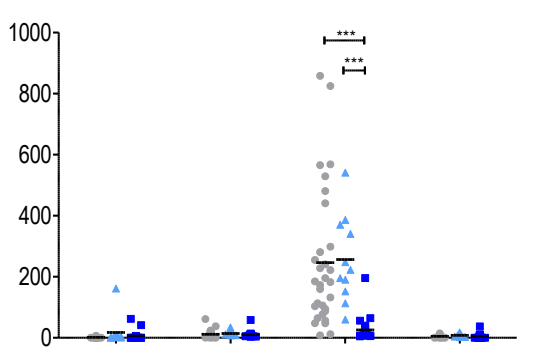

CXCL10

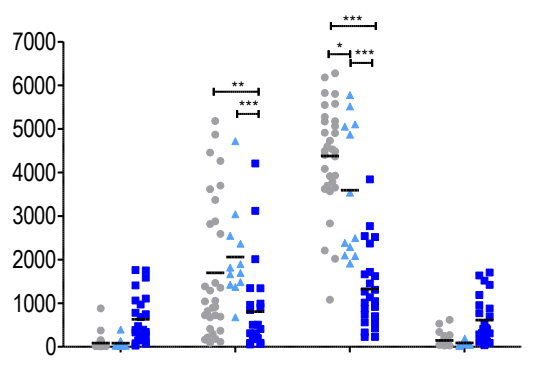

IFNy

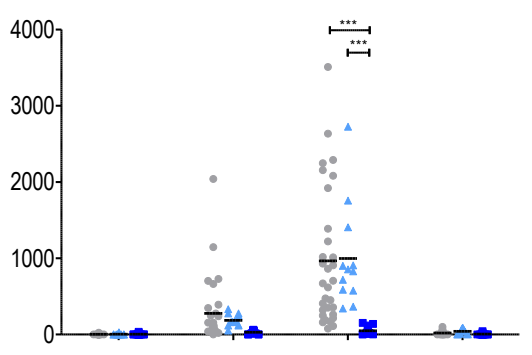

IL10

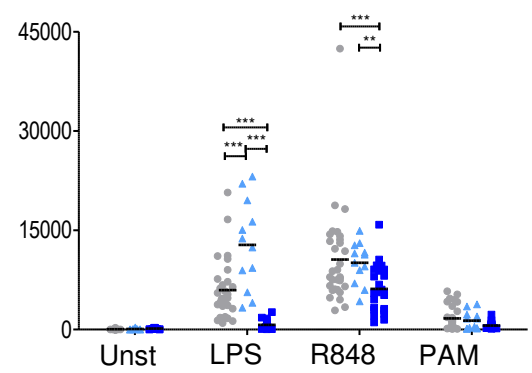

Fig.1. Reduced levels of cytokines in severe patients during acute infection. Dosage of cytokines and chemokines in the supernatant of whole blood culture after stimulation for $24 \mathrm{~h}$ in controls $(n=32)$, mild $(n=11)$ and severe $(n=24)$ patients during acute infection. A two-way ANOVA test was performed to examine the statistical differences of each cytokine per group and stimulation, followed by Bonferroni posttests.

${ }^{\star} p<0.05,{ }^{* \star} p<0.01,{ }^{* \star \star} p<0.001,{ }^{* \star \star *} p<0.0001$ 
A

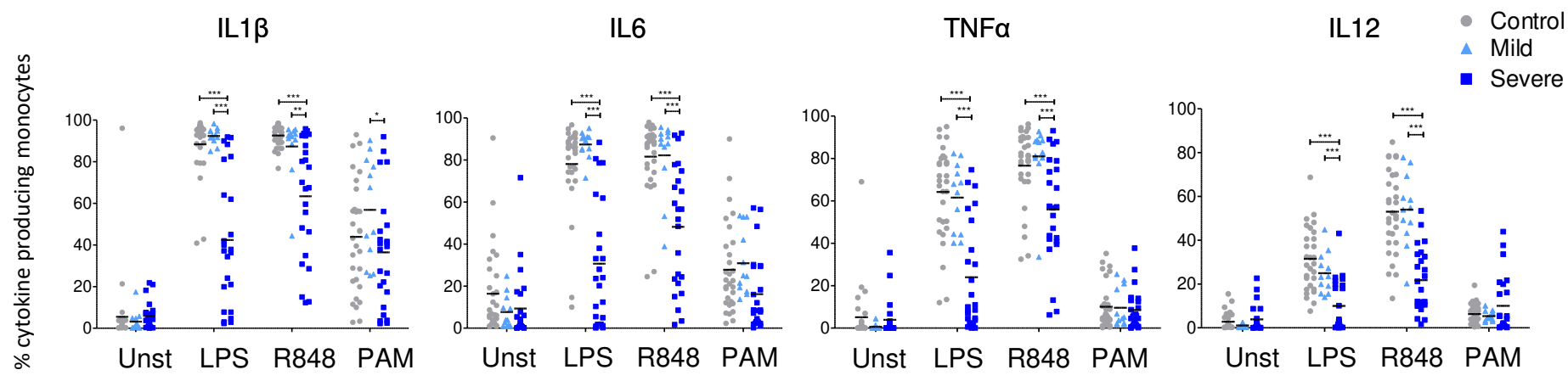

$\mathrm{B}$
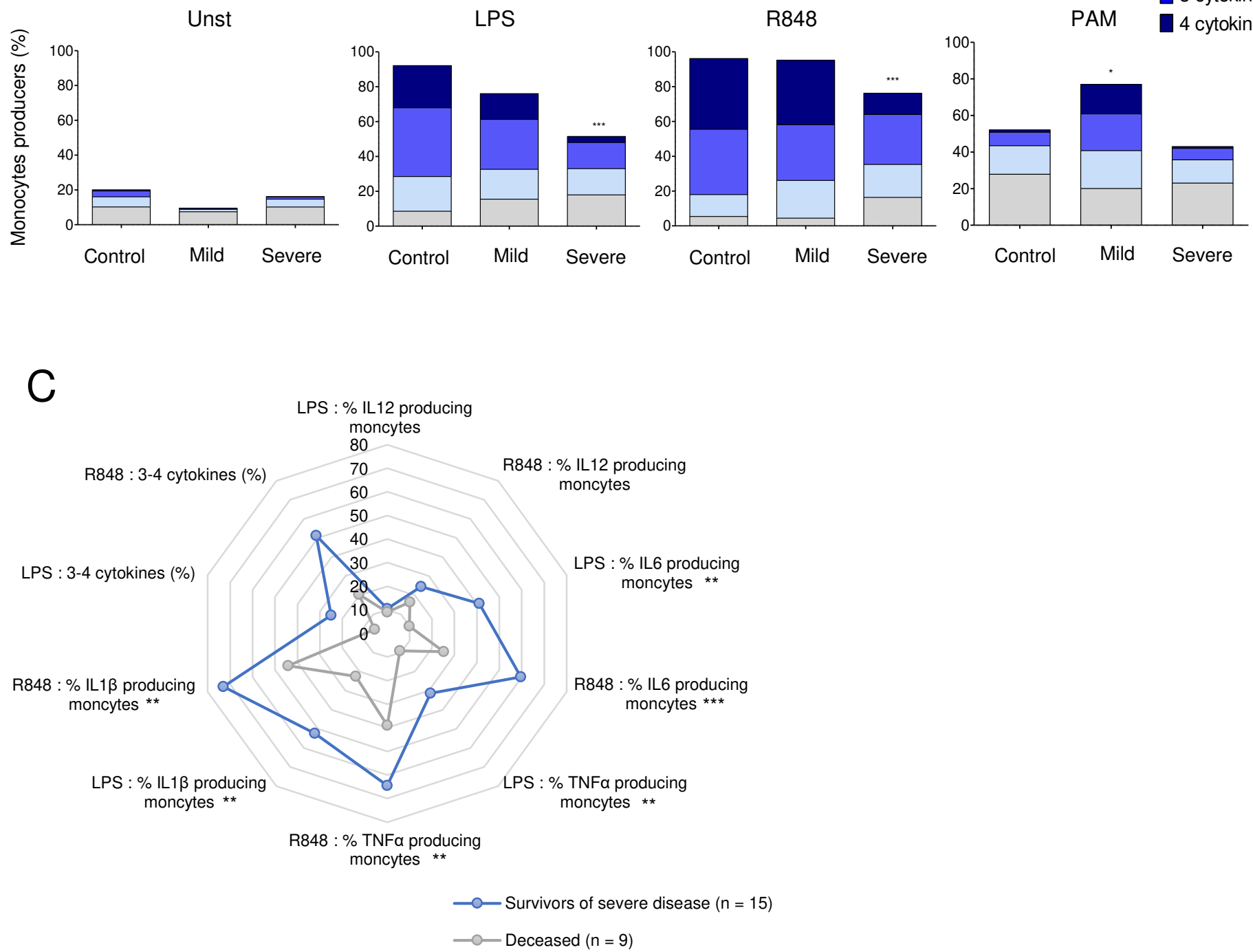

Fig.2. Cytokine production by CD14+ monocytes is impaired in response to various PAMPs in patients with acute severe COVID-19. (A) Flow cytometry analysis of intracellular production of cytokines by CD14+ monocytes upon $6 \mathrm{~h}$ stimulation of whole blood from controls $(n=32)$, mild $(n=11)$ and severe $(n=24)$ patients during acute infection. A two-way ANOVA test was performed to examine the statistical differences of each cytokine/monocyte per group and stimulation, followed by Bonferroni posttests. (B) Measure of the ability of monocytes to produce up to 4 cytokines among TNF- $\alpha$, IL-6, IL-12p40 and IL-1 $\beta$ simultaneously (polyfunctionality) upon distinct stimulations among acute patients. Kruskal-Wallis test was performed to examine the statistical differences in the ability to produce 3 or 4 cytokines simultaneously per group, followed by Dunn's correction for multiple testing, statistical difference is expressed compared to controls. (C) Radar chart of cytokines production and polyfunctionality upon LPS and R848 stimulation, according to the outcome among severe patients. 
IL $1 \beta$

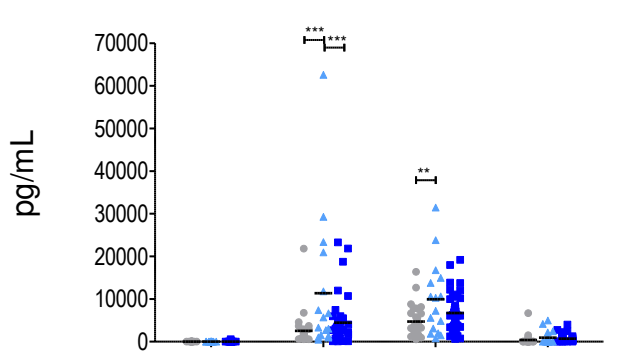

IL6

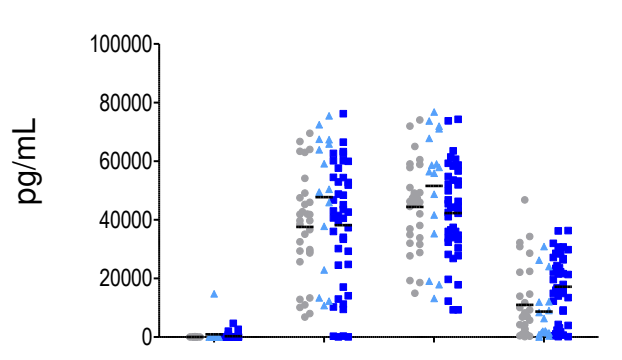

TNFa

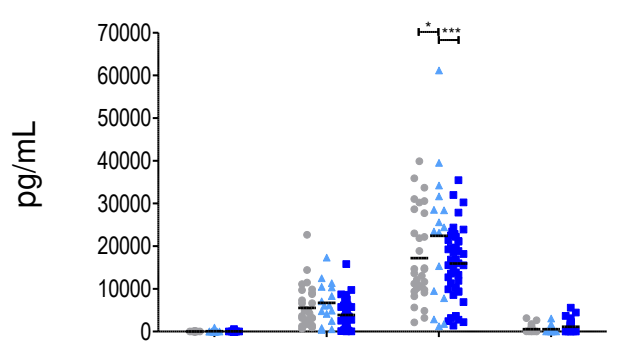

IL12p70
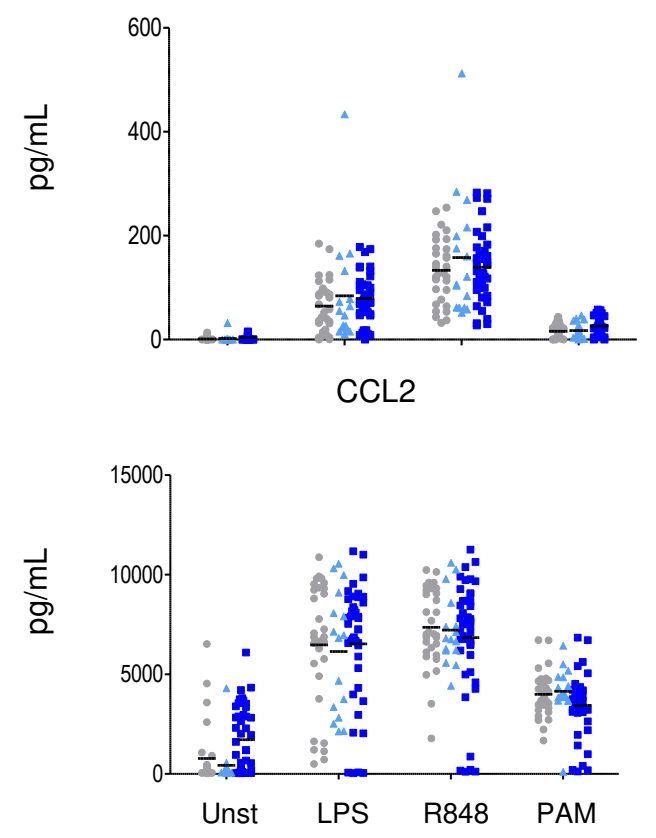

IFN $\beta$

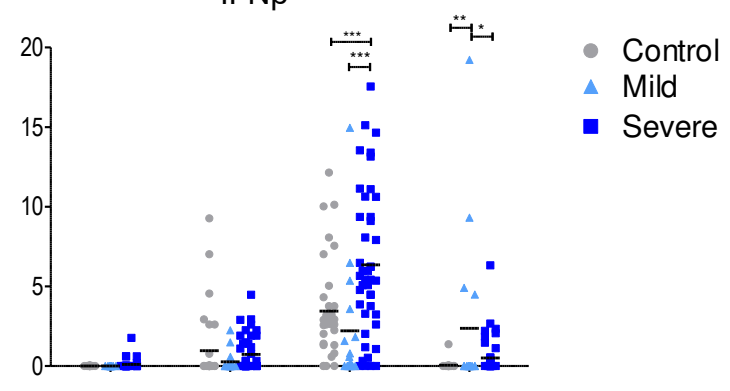

IFNa

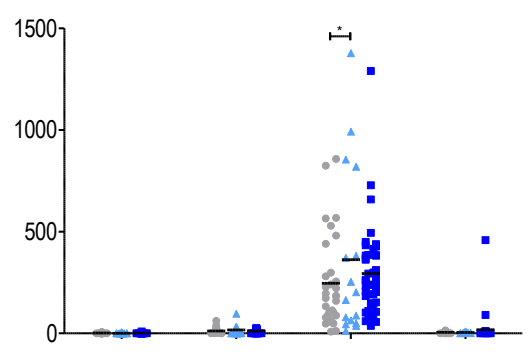

CXCL10

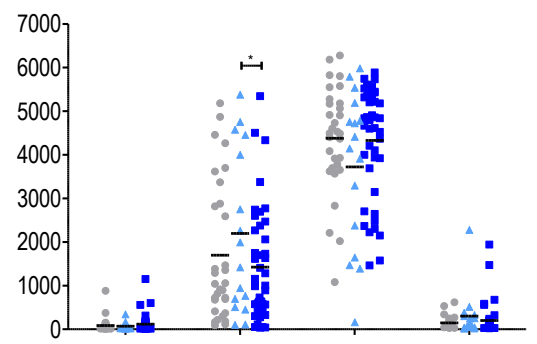

IFNY

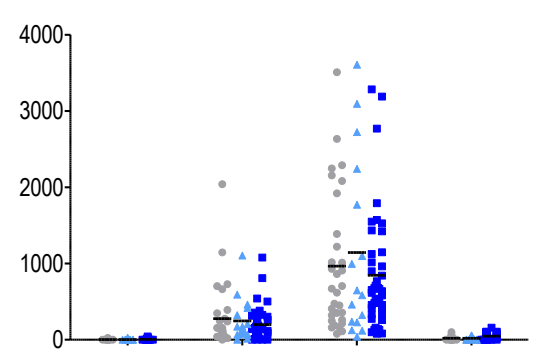

IL10

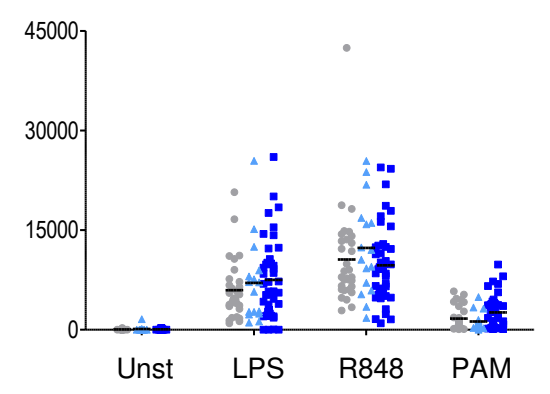

Fig.3. Normalization of TLR-elicited cytokine levels in whole blood from convalescent patients. Dosage of cytokines and chemokines in the supernatant of whole blood culture after stimulation for $24 \mathrm{~h}$ in controls $(n=32)$, mild $(n=19)$ and severe $(n=43)$ patients during recovery phase. A two-way ANOVA test was performed to examine the statistical differences of each cytokine/monocyte per group and stimulation, followed by Bonferroni posttests.

${ }^{*} p<0.05,{ }^{* *} p<0.01,{ }^{* * *} p<0.001,{ }^{* * * *} p<0.0001$ 

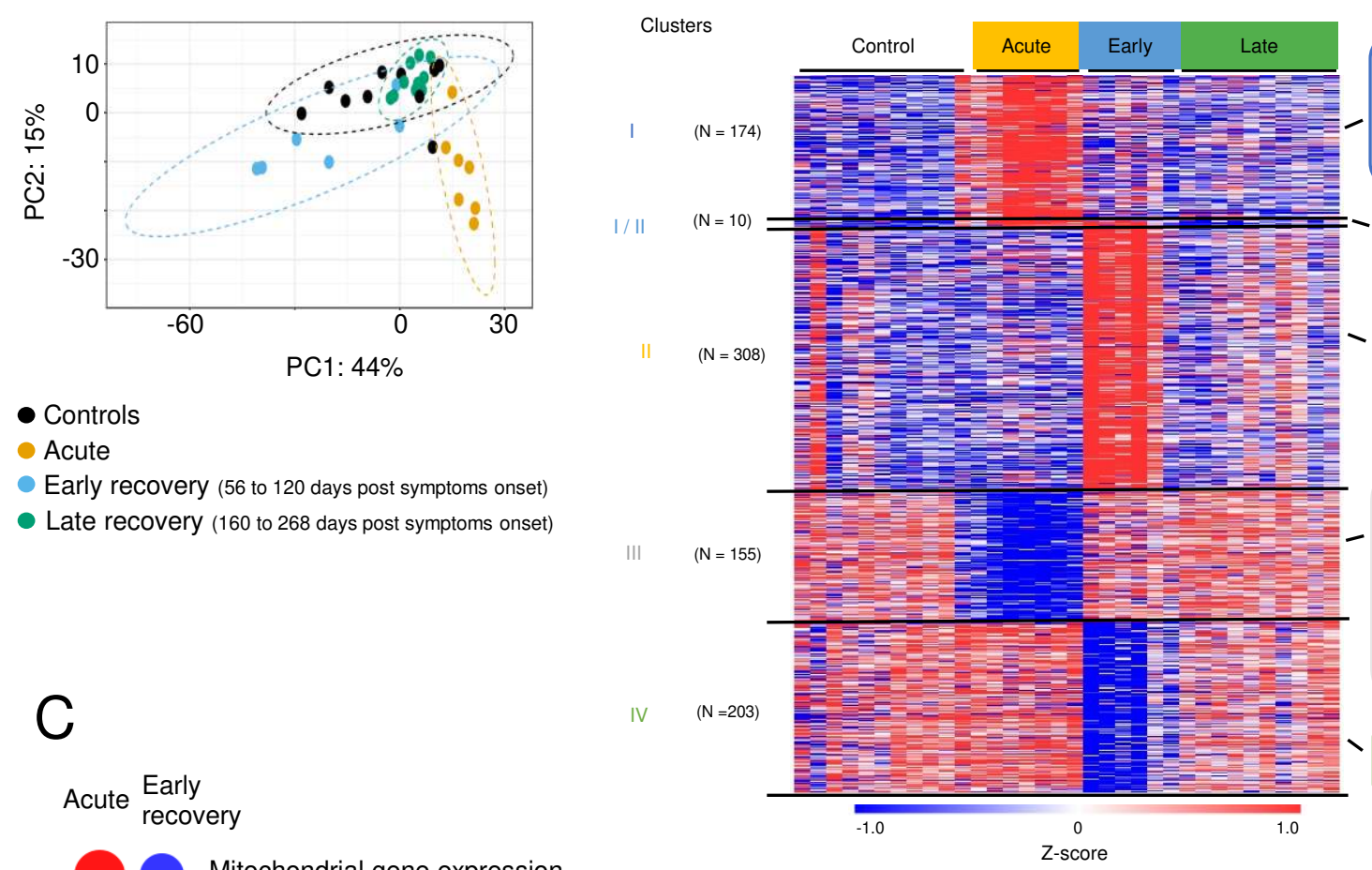

Representative genes

- Controls

- Acute

- Early recovery (56 to 120 days post symptoms onset)

- Late recovery (160 to 268 days post symptoms onset)

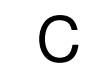

$$
\text { Acute Early }
$$

Mitochondrial gene expression

Fatty acid derivative metabolism

Regulation of cellular amino acid metabolism

Glycosylation

Regulation of type I IFN mediated signaling pathway

Viral gene expression

MHC II protein complex binding

Positive regulation of intrinsic apoptotic signaling

Positive regulation of cell killing

Positive regulation of wound healing

Chemokine activity

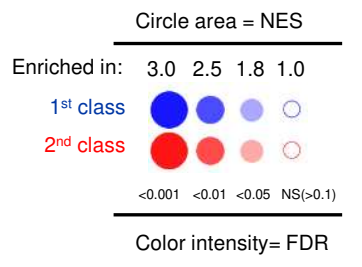

Covid-19

Associated monocytes clusters

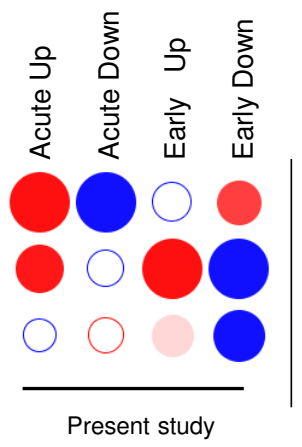

Disease severity HLA-DR ${ }^{\prime \circ}$
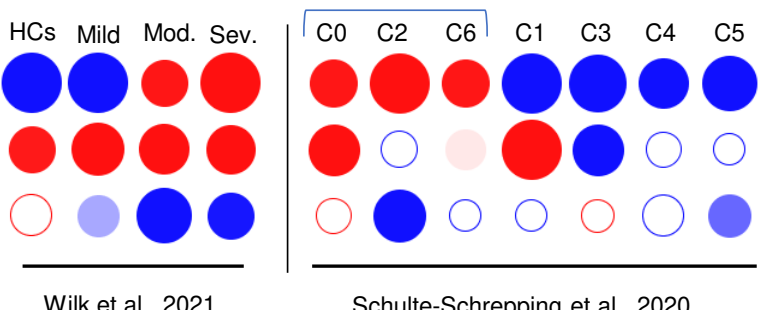

Schulte-Schrepping et al., 2020

Present study

\begin{tabular}{|c|c|c|}
\hline $\begin{array}{l}\text { DHCR24 } \\
\text { HPGD } \\
\text { SCDDHFR } \\
\text { S100A8 } \\
\text { VSIG4 } \\
\text { CD163 } \\
\text { FLT3 }\end{array}$ & $\begin{array}{l}\text { ACSL1 } \\
\text { MSMO } \\
\text { MPO } \\
\text { CLU } \\
\text { FCGR1A } \\
\text { IFI27 }\end{array}$ & $\begin{array}{l}\text { ALOX15B } \\
\text { LDHA1 } \\
\text { S100A12 } \\
\text { HMGB2 } \\
\text { LAIR1 } \\
\text { IL1R2 }\end{array}$ \\
\hline SQLE & SPRY2 & PPARG \\
\hline $\begin{array}{l}\text { CCL2 } \\
\text { CXCL1 } \\
\text { TNFRSF12A } \\
\text { TNFSF14 } \\
\text { EIF2AK3 } \\
\text { JARID2 } \\
\text { IL1RN } \\
\text { HAVCR2 }\end{array}$ & $\begin{array}{l}\text { CCL4 } \\
\text { CXCL16 } \\
\text { CSF1 } \\
\text { TNFSF8 } \\
\text { FOXO3 } \\
\text { IRF2BP2 } \\
\text { DOT1 } \\
\text { MAFF }\end{array}$ & $\begin{array}{l}\text { CCL7 } \\
\text { CXCL2 } \\
\text { IL1R1 } \\
\text { DDIT3 } \\
\text { HMGA1 } \\
\text { IRAK2 } \\
\text { SOCS6L } \\
\text { MAFB }\end{array}$ \\
\hline $\begin{array}{l}\text { IL1B } \\
\text { C3 } \\
\text { HLA-DMA } \\
\text { HLA-DMB } \\
\text { HLA-DQA1 } \\
\text { HLA-DQA2 } \\
\text { HLA-DPA1 } \\
\text { HLA-DOA } \\
\text { HLA-DRA } \\
\text { HLA-DRB1 } \\
\text { ATF3 }\end{array}$ & $\begin{array}{l}\text { GPR183 } \\
\text { FTH1 } \\
\text { CLEC10A } \\
\text { MAP3K14 } \\
\text { CD4 } \\
\text { OSMa } \\
\text { CLEC4A } \\
\text { SRCCAMK2D } \\
\text { NRP1 } \\
\text { NR4A1 } \\
\text { JUNB }\end{array}$ & $\begin{array}{l}\text { CIITA } \\
\text { KLF12 } \\
\text { CSF1R } \\
\text { GPR183 } \\
\text { VEGFA } \\
\text { PTGER4 } \\
\text { CSF1R } \\
\text { KLF11 } \\
\text { PDGFC } \\
\text { NFE2L3 } \\
\text { NFKB2 }\end{array}$ \\
\hline $\begin{array}{l}\text { PLAG1 } \\
\text { HIST1H3C }\end{array}$ & $\begin{array}{l}\text { METTL17 } \\
\text { ZNF490 }\end{array}$ & $A P A F 1$ \\
\hline
\end{tabular}

Wilk et al., 2021 $\begin{array}{ll}\text { PLAG1 } & \text { METTL17 } \\ \text { HIST1H3C } & \text { ZNF490 }\end{array}$

Control vs Acute

Control vs Early recovery

Control vs Late recovery

Fig.5 : Distinct transcriptomic profiles of monocytes during acute and recovery phase. (A) PCA plot representing the distinct clusters based on transcriptional profiles of monocytes from controls $(n=11)$, acute infection $(n=7)$, "early recovery" $(n=6)$ and "late recovery" phase $(n=10)$. (B) Heatmap of differential genes for controls $(n=11)$, acute infection $(n=7)$, "early recovery" ( $n=6)$ and "late recovery" phase $(n=10)$, representative genes and interesting pathways were selected. (C) BubbleGUM GSEA map established from available gene sets in monocytes during Covid-19 or sepsis. For each gene set, origin of the data set of up- (red) and down- (blue) regulated genes are indicated. The panel summarizes the NES and FDR parameters.

FDR, false discovery rate; GSEA, gene set enrichment analysis; HCs, healthy controls ; NES, normalized enrichment score; PCA, principal component analysis; Mod., moderate ; Sev., severe 

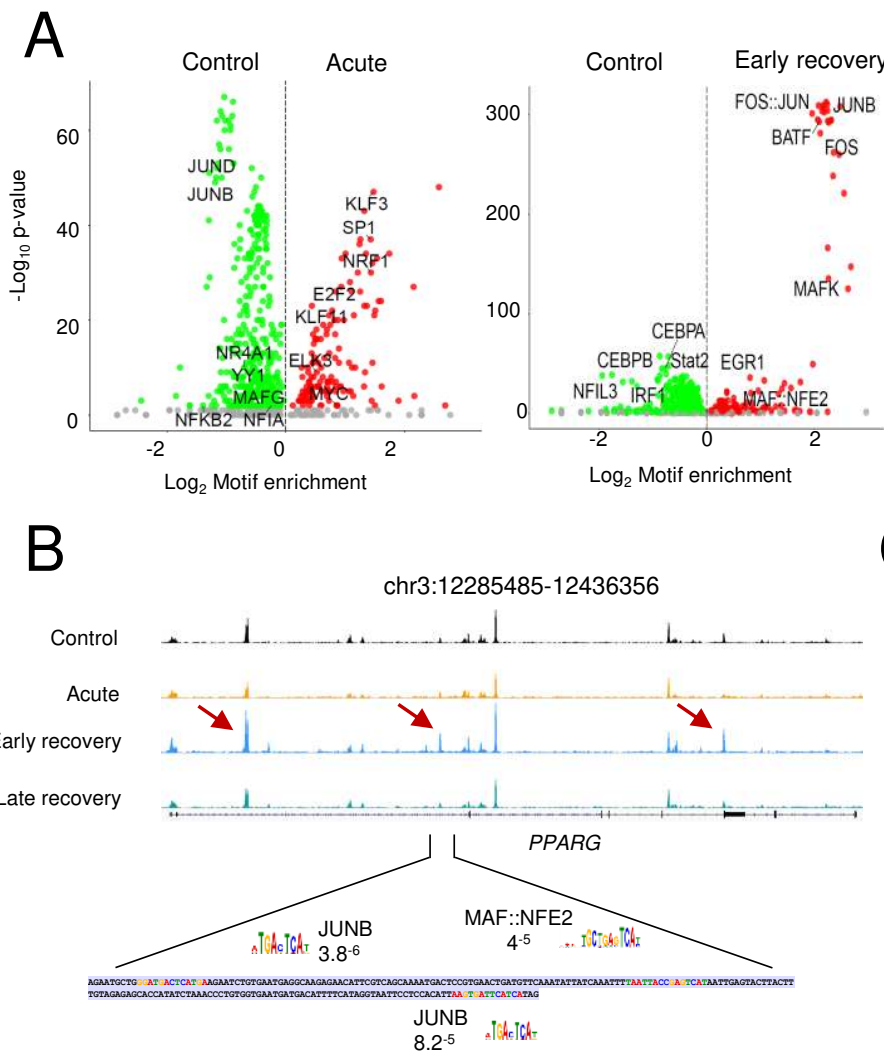

chr3:12285485-12436356

$\mathrm{D}$
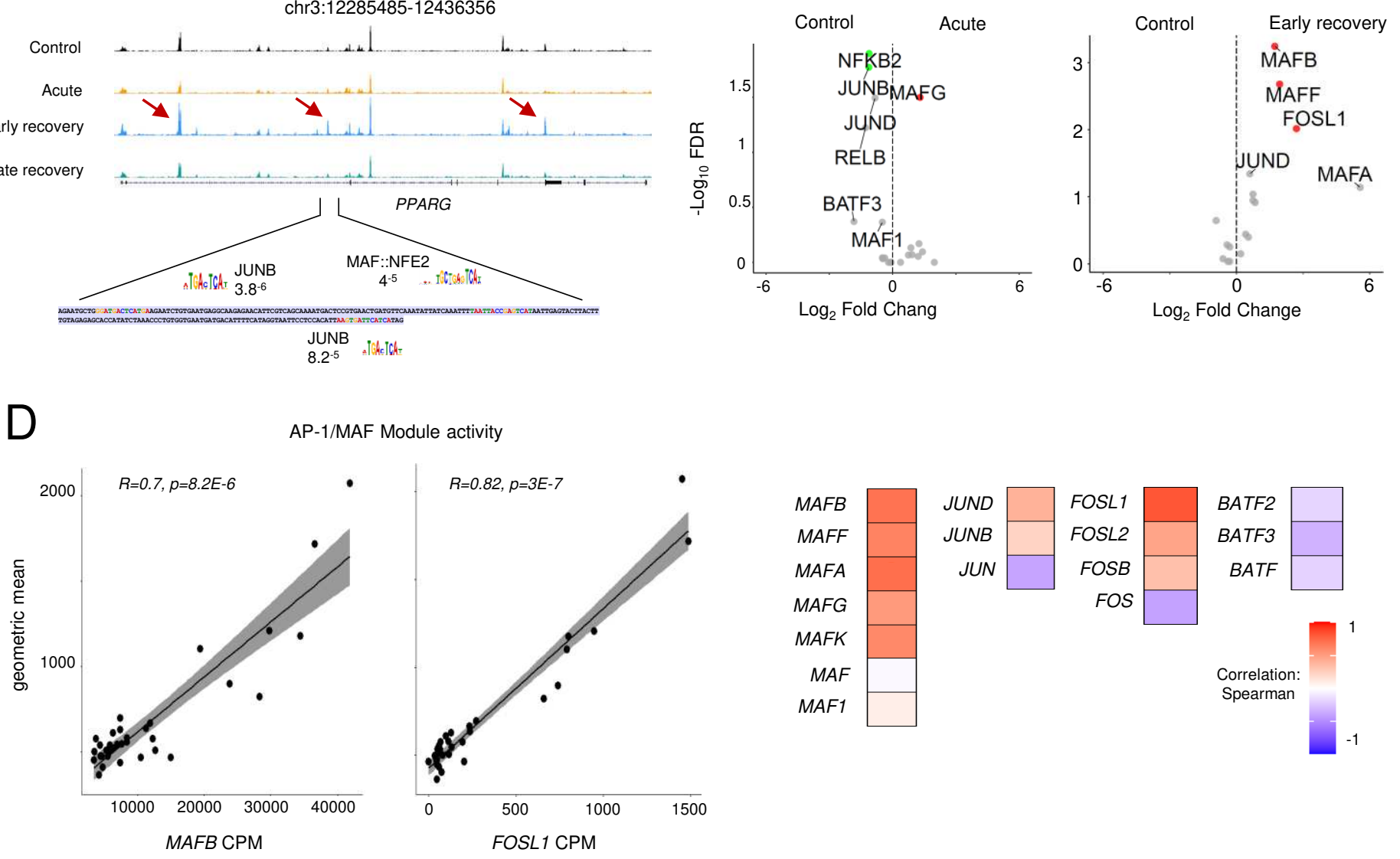

Early recovery

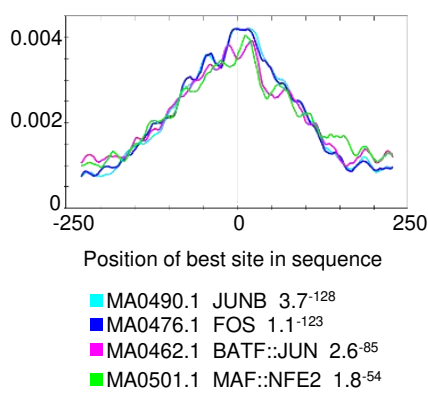
opened regions

\section{C}

DA0050.2 IRF1 $3.7^{-10}$

MA0517.1 STAT1::STAT2 3.8

MA0517.1 STAT1::STA
MA0107.1 RELA $2.2^{-7}$

E

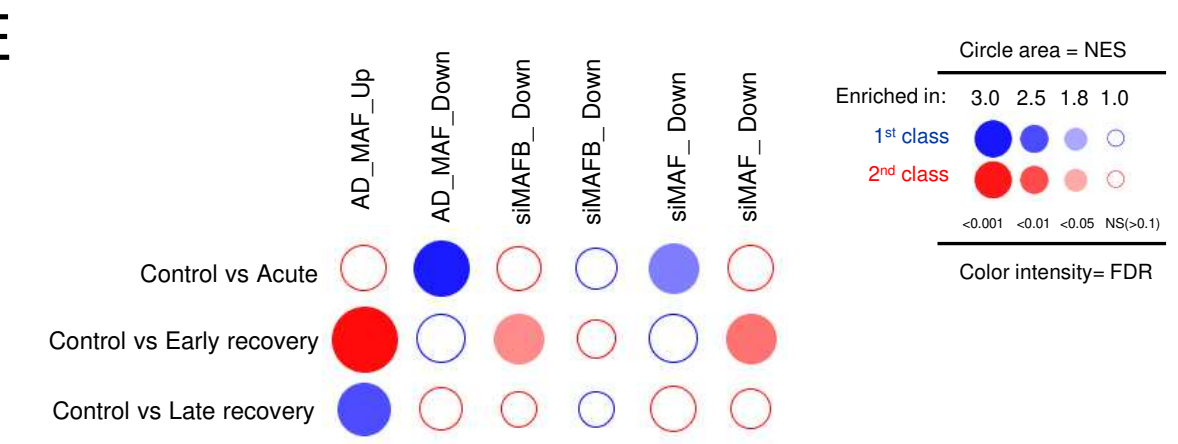

Fig.7 : Motifs associated with differentially accessible regions in monocytes from Covid-19 patients. (A) Ciiider analysis of putative transcription factor motifs enrichment (left) and representative motifs centrality (right) on differentially opened and closed regions in acute and convalescing patients. (B) Representative ATAC-Seq tracks at the PPARG locus. Peaks with increased accessibility in early recovery patients are indicated by a red arrow. Sequence of the highlighted region and the location of JUN and MAF motifs with their p-value are indicated (C) Volcano plot showing changes in expression of AP-1, NF-KB and MAF genes in acute (right) or early recovery patients (left) in comparison to controls. (D) Correlation between the mRNA expression of the indicated transcription factor and the AP-1/MAF module activity in the whole data set. Representative plots for MAFB and FOSL1 are shown. Spearman correlation coefficients for each gene are represented in a heatmap (right). (E) BubbleGUM GSEA of datasets from controls, early and late recovery patients. Publicly available gene sets were obtained from macrophages infected by MAF-expressing Adenovirus (24) or treated with siRNA targeting either MafB or MAF (42). The panel summarizes the normalized enrichment score (NES) and false discovery rate $(F D R)$ parameters.

FDR, false discovery rate ; GSEA, gene set enrichment analysis ; NES, normalized enrichment score 


\section{Supplementary Files}

This is a list of supplementary files associated with this preprint. Click to download.

- BraunsetalSupFigs.pdf 